\title{
ON FALCONER'S FORMULA FOR THE GENERALIZED RÉNYI DIMENSION OF A SELF-AFFINE MEASURE
}

\author{
Ian D. Morris \\ University of Surrey, Department of Mathematics \\ Guildford GU2 7XH, U.K.; i.morris@surrey.ac.uk
}

\begin{abstract}
We investigate a formula of Falconer which describes the typical value of the generalised Rényi dimension, or generalised $q$-dimension, of a self-affine measure in terms of the linear components of the affinities. We show that in contrast to a related formula for the Hausdorff dimension of a typical self-affine set, the value of the generalised $q$-dimension predicted by Falconer's formula varies discontinuously as the linear parts of the affinities are changed. Conditionally on a conjecture of Bochi and Fayad, we show that the value predicted by this formula for pairs of two-dimensional affine transformations is discontinuous on a set of positive Lebesgue measure. These discontinuities derive from discontinuities of the lower spectral radius which were previously observed by the author and Bochi.
\end{abstract}

\section{Introduction}

If $\mathrm{T}:=\left(T_{1}, \ldots, T_{N}\right)$ is a finite collection of transformations of a complete metric space $X$, and each $T_{i}$ is a contraction in the sense that for some $\lambda<1$ one has $d\left(T_{i} x, T_{i} y\right) \leq \lambda d(x, y)$ for all $x, y \in X$, it is well-known that there exists a unique nonempty compact set $Z_{\mathrm{T}} \subseteq X$ such that

$$
Z_{\mathrm{T}}=\bigcup_{i=1}^{N} T_{i}\left(Z_{\mathrm{T}}\right)
$$

see for example $[11,18]$. The case in which $X=\mathbf{R}^{d}$ and the transformations $T_{i}$ are affine - in which case $Z_{\mathrm{T}}$ is called a self-affine set-has been the subject of intense study over the last few decades (for a recent survey we note [13]).

Various notions of fractal dimension have been investigated for both general and special classes of self-affine set. In certain special cases where the linear parts of the affinities preserve or permute the horizontal and vertical axes of $\mathbf{R}^{2}$, explicit formulæ for the Hausdorff dimension and box dimension exist (see e.g. [3, 5, 16, 21, 23]). In the context of general self-affine sets, a landmark result of Falconer [8] established the Hausdorff and box dimensions of "typical" self-affine sets in a sense which we now describe. Let $M_{d}(\mathbf{R})$ denote the set of all $d \times d$ real matrices, and recall that for $A \in M_{d}(\mathbf{R})$ we define the singular values of $A$, denoted $\sigma_{1}(A), \ldots, \sigma_{d}(A)$, to be the non-negative square roots of the eigenvalues of the positive semi-definite matrix $A^{*} A$, listed in decreasing order. Let us define a function $\varphi:(0,+\infty) \times M_{d}(\mathbf{R}) \rightarrow[0,+\infty)$ by

$$
\varphi^{s}(A):= \begin{cases}\sigma_{1}(A) \cdots \sigma_{k}(A) \sigma_{k+1}(A)^{s-k}, & k \leq s \leq k+1 \leq d, \\ |\operatorname{det} A|^{\frac{s}{d}}, & s \geq d,\end{cases}
$$

https://doi.org/10.5186/aasfm.2017.4214

2010 Mathematics Subject Classification: Primary 28A80.

Key words: Lower spectral radius, self-affine measure, self-affine set, q-dimension, Rényi dimension. 
and for every $A_{1}, \ldots, A_{N}$ in the open unit ball of $M_{d}(\mathbf{R})$ define the affinity dimension or singularity dimension of $\left(A_{1}, \ldots, A_{N}\right)$ to be the quantity

$$
\mathfrak{s}\left(A_{1}, \ldots, A_{N}\right):=\inf \left\{s>0: \sum_{n=1}^{\infty} \sum_{i_{1}, \ldots, i_{n}=1}^{N} \varphi^{s}\left(A_{i_{1}} \cdots A_{i_{n}}\right)<\infty\right\} .
$$

Falconer showed that for any fixed invertible $d \times d$ matrices $A_{1}, \ldots, A_{N}$ with Euclidean norm strictly less than $\frac{1}{3}$, for Lebesgue almost all $v_{1}, \ldots, v_{N} \in \mathbf{R}^{d}$ the self-affine set $Z_{\mathrm{T}}$ associated to the collection of affine transformations $\mathrm{T}:=\left(T_{1}, \ldots, T_{N}\right)$ defined by $T_{i} x:=A_{i} x+v_{i}$ satisfies

$$
\operatorname{dim}_{H}\left(Z_{\mathrm{T}}\right)=\operatorname{dim}_{B}\left(Z_{\mathrm{T}}\right)=\min \left\{\mathfrak{s}\left(A_{1}, \ldots, A_{N}\right), d\right\},
$$

see [8]; the bound on the norm was subsequently relaxed to $\frac{1}{2}$ by B. Solomyak [25], and to 1 by Jordan, Simon and Pollicott for a notion of "almost self-affine set" which incorporates additional random translations [19]. While it is well-known that the Hausdorff dimension of $Z_{\mathrm{T}}$ can fail to depend continuously on the affinites $T_{1}, \ldots, T_{N}$ (see e.g. [11, Example 9.10]), it was recently shown by Feng and Shmerkin in [15] that the affinity dimension $\mathfrak{s}$ is a continuous function of $\left(A_{1}, \ldots, A_{N}\right)$. An alternative proof of this statement was subsequently given by the author [24].

In this article we will focus not on self-affine sets but on self-affine measures. A Borel probability measure $\mu$ on $\mathbf{R}^{d}$ is called self-affine if there exist a probability vector $\mathbf{p}=\left(p_{1}, \ldots, p_{N}\right)$ and a collection of affinities $\mathbf{T}=\left(T_{1}, \ldots, T_{N}\right)$ such that

$$
\mu(A)=\sum_{i=1}^{N} p_{i} \mu\left(T_{i}^{-1} A\right)
$$

for every Borel set $A \subseteq \mathbf{R}^{d}$. If $T_{1}, \ldots, T_{N}$ are contractions then for each probability vector $\mathrm{p}$ with all probabilities nonzero there exists a unique Borel probability measure $\mu_{\mathrm{p}, \mathrm{T}}$ satisfying the above functional equation (see e.g. [9, Theorem 2.8]), and the support of that measure is equal to the associated self-affine set $Z_{\mathrm{T}}$. In this article our interest is in the generalised q-dimension or generalised Rényi dimension of a self-affine measure, which is defined as follows. For each $r>0$ let $\mathcal{M}_{r}$ denote the set of all $r$-mesh cubes on $\mathbf{R}^{d}$, that is, the set of all $d$-dimensional cubes of the form $\left[j_{1} r,\left(j_{1}+1\right) r\right) \times\left[j_{2} r,\left(j_{2}+1\right) r\right) \times \cdots \times\left[j_{d} r,\left(j_{d}+1\right) r\right)$ where $j_{1}, \ldots, j_{d} \in \mathbf{Z}$. For $q>1$ we define

$$
M_{r}(q, \mu):=\sum_{C \in \mathcal{M}_{r}} \mu(C)^{q}
$$

for every $r>0$, and

$$
\underline{D}_{q}(\mu):=\liminf _{r \rightarrow 0} \frac{\log M_{r}(q, \mu)}{(q-1) \log r}, \quad \bar{D}_{q}(\mu):=\limsup _{r \rightarrow 0} \frac{\log M_{r}(q, \mu)}{(q-1) \log r} .
$$

If $\underline{D}_{q}(\mu)$ and $\bar{D}_{q}(\mu)$ are equal then we define the generalised $q$-dimension of $\mu$ to be their common value and denote this by $D_{q}(\mu)$. For $q>1$ the generalised $q$ dimension admits an alternative expression as a limit of certain integrals [22]. In [10, Theorem 6.2], Falconer characterised the generalised Rényi dimensions of typical selfaffine measures in a similar manner to his earlier characterisation of the Hausdorff and box dimensions of typical self-affine sets:

Theorem 1.1. (Falconer) Let $\left(A_{1}, \ldots, A_{N}\right)$ be invertible linear transformations of $\mathbf{R}^{d}$ such that $\left\|A_{i}\right\|<\frac{1}{2}$ for every $i$, let $\mathrm{p}=\left(p_{1}, \ldots, p_{N}\right)$ be a probability vector with 
all entries nonzero, and for each $q>1$ define $\mathfrak{r}_{q}\left(A_{1}, \ldots, A_{N}, \mathrm{p}\right)$ to be the quantity

$$
\sup \left\{s>0: \sum_{n=1}^{\infty} \sum_{i_{1}, \ldots, i_{n}=1}^{N} \varphi^{s}\left(A_{i_{1}} \cdots A_{i_{n}}\right)^{1-q} p_{i_{1}}^{q} \cdots p_{i_{n}}^{q}<\infty\right\} .
$$

If $1<q \leq 2$ then for Lebesgue-almost-every $\left(v_{1}, \ldots, v_{N}\right) \in \mathbf{R}^{N d}$ the self-affine measure $\mu_{\mathrm{p}, \mathrm{T}}$ corresponding to the transformations $T_{i} x:=A_{i} x+v_{i}$ and the probability vector $\mathrm{p}$ satisfies $D_{q}\left(\mu_{\mathrm{p}, \mathrm{T}}\right)=\min \left\{\mathfrak{r}_{q}\left(A_{1}, \ldots, A_{N}, \mathrm{p}\right), d\right\}$.

In the later article [12] this result was extended to a more general class of almost self-affine measures, under the weaker hypotheses $\max \left\|A_{i}\right\|<1$ and $q>1$ but requiring randomised translations in a similar manner to [19]. An alternative extension of this result, which allows $q \in[1, Q]$ for certain values of $Q>2$, was recently obtained by Barral and Feng $[4, \S 6]$. In view of the recent work of Feng and Shmerkin [15] on the continuity of the formula $\mathfrak{s}\left(A_{1}, \ldots, A_{N}\right)$ for the typical dimension of a self-affine set, it is natural to ask whether the formula $\mathfrak{r}_{q}\left(A_{1}, \ldots, A_{N}, \mathrm{p}\right)$ for the typical dimension of a self-affine measure is also continuous with respect to changes in the matrices $A_{1}, \ldots, A_{N}$. The purpose of this article is to answer this question negatively.

In order to state our results we require an additional definition. Let us say that a pair of matrices $\left(A_{1}, A_{2}\right)$ is $(c, \varepsilon, \lambda)$-resistant if it has the following property: for all choices of $i_{1}, \ldots, i_{n} \in\{1,2\}$ such that at most $\varepsilon n$ of the integers $i_{k}$ are equal to 2 , we have $\left\|A_{i_{1}} \cdots A_{i_{n}}\right\| \geq c \lambda^{n}$. We will say that $\left(A_{1}, A_{2}\right)$ resists impurities, or more simply is resistant, if it is $(c, \varepsilon, \lambda)$-resistant for some $c, \varepsilon>0$ and some $\lambda>1$. We recall the following conjecture of Bochi and Fayad [6]:

Conjecture 1.2. (Bochi-Fayad Conjecture) Let $\mathcal{H}$ denote the set of all $2 \times 2$ real matrices with unit determinant and unequal real eigenvalues, and let $\mathcal{E}$ denote the set of all $2 \times 2$ real matrices with unit determinant and non-real eigenvalues. Then the set of resistant pairs $\left(A_{1}, A_{2}\right) \in \mathcal{H} \times \mathcal{E}$ has full Lebesgue measure.

Some partial results in the direction of Conjecture 1.2 may be found in $[1,2,14]$. Bonatti has constructed explicit examples of resistant pairs in which $A_{2}$ is a rational rotation, and these examples are described in [7].

When investigating the discontinuities of $\mathfrak{r}_{q}$ we will focus on the situation in which $\left(A_{1}, \ldots, A_{N}\right)$ is a pair of real matrices of dimension two. In this case the probability vector $\mathrm{p}=\left(p_{1}, p_{2}\right)$ has the form $(p, 1-p)$ for some real number $p \in(0,1)$, and in view of this we shall simply write $\mathfrak{r}_{q}\left(A_{1}, A_{2}, p\right)$ in place of the value $\mathfrak{r}_{q}\left(A_{1}, A_{2},(p, 1-p)\right)$ defined previously. We prove:

Theorem 1.3. The function $\mathfrak{r}$ admits the following discontinuities:

(i) Let $q>1, p \in(0,1)$ and $0<\delta<\lambda<\frac{1}{2}$, and suppose that $\delta$ is small enough that

$$
\frac{\log \min \left\{p^{q},(1-p)^{q}\right\}}{\log \sqrt{\lambda \delta}}<\frac{\log \left(p^{q}+(1-p)^{q}\right)}{\log \lambda} .
$$

Then the function $\left(A_{1}, A_{2}\right) \mapsto \mathfrak{r}_{q}\left(A_{1}, A_{2}, p\right)$ is discontinuous at the pair

$$
A_{1}:=\left(\begin{array}{cc}
\lambda & 0 \\
0 & \delta
\end{array}\right), \quad A_{2}:=\left(\begin{array}{cc}
\lambda & 0 \\
0 & \lambda
\end{array}\right)
$$

(ii) If the Bochi-Fayad Conjecture is true then there exists a set $X \subseteq M_{2}(\mathbf{R})^{2}$ with positive Lebesgue measure with the following properties: $\left\|A_{1}\right\|,\left\|A_{2}\right\|<\frac{1}{2}$ for all $\left(A_{1}, A_{2}\right) \in X$, and there exists $Q>1$ such that for all $p \in\left[\frac{1}{2}, 1\right)$ and 
$q>Q$, the function $\left(A_{1}, A_{2}\right) \mapsto \mathfrak{r}_{q}\left(A_{1}, A_{2}, p\right)$ is discontinuous at every point of $X$.

Remark. The reader will see from the proofs below that Theorem 1.3(i) in fact has the following more precise statement: if $R_{\theta}$ denotes the matrix corresponding to rotation about the origin through angle $\theta$, then

$$
\liminf _{\theta \rightarrow 0} \mathfrak{r}_{q}\left(A_{1}, \lambda R_{\theta}, p\right)<\mathfrak{r}_{q}\left(A_{1}, A_{2}, p\right) .
$$

Clearly the case of $p<\frac{1}{2}$ may also be considered in (ii) by interchanging the rôles of the matrices $A_{1}$ and $A_{2}$.

It would be of interest to remove the restriction on $q$ in (ii) so as to bring that statement into line with (i). Unfortunately the Bochi-Fayad Conjecture does not seem to be a sufficiently strong statement to allow us to deduce that $\left(A_{1}, A_{2}\right) \mapsto$ $\mathfrak{r}_{q}\left(A_{1}, A_{2}, p\right)$ has a positive-measure set of discontinuities for every $p \in(0,1)$ and $q>1$. We nonetheless conjecture that this map has a positive-measure set of discontinuities for all such $p$ and $q$, and hope that whatever methods may be employed to prove the Bochi-Fayad Conjecture will also suffice to establish the discontinuity of $\mathfrak{r}_{q}(\cdot, \cdot, p)$ on a set of positive measure for all $q>1$ and $p \in(0,1)$.

\section{Proof of Theorem 1.3}

For $p \in(0,1), q>1, s>0$ and invertible matrices $A_{1}, A_{2} \in M_{2}(\mathbf{R})$, let us define $p_{1}:=p$ and $p_{2}:=(1-p)$, and write

$$
\mathbf{R}_{q}\left(A_{1}, A_{2}, p, s\right):=\lim _{n \rightarrow \infty} \frac{1}{n} \log \left(\sum_{i_{1}, \ldots, i_{n}=1}^{N} \varphi^{s}\left(A_{i_{1}} \cdots A_{i_{n}}\right)^{1-q} p_{i_{1}}^{q} \cdots p_{i_{n}}^{q}\right) .
$$

We note:

Lemma 2.1. For fixed invertible matrices $A_{1}, A_{2} \in M_{d}(\mathbf{R})$ such that $\left\|A_{1}\right\|$, $\left\|A_{2}\right\|<1$, fixed $q>1$ and fixed $p \in(0,1)$ the function $\mathbf{R}_{q}\left(A_{1}, A_{2}, p, \cdot\right):(0,+\infty) \rightarrow \mathbf{R}$ is well-defined and strictly increasing.

Proof. It is well-known that $\varphi^{s}(A B) \leq \varphi^{s}(A) \varphi^{s}(B)$ for all $s>0$ and $A, B \in$ $M_{d}(\mathbf{R})$, see for example [8, Lemma 2.1]; since the proof is brief we include it. For $s \geq d$ the result is trivial, and for $k \leq s<k+1, k=0, \ldots, d-1$ we have

$$
\begin{aligned}
\varphi^{s}(A B) & =\left(\sigma_{1}(A B) \cdots \sigma_{k+1}(A B)\right)^{s-k}\left(\sigma_{1}(A B) \cdots \sigma_{k}(A B)\right)^{k+1-s} \\
& =\left\|\wedge^{k+1}(A B)\right\|^{s-k}\left\|\wedge^{k}(A B)\right\|^{k+1-s} \\
& \leq\left\|\wedge^{k+1} A\right\|^{s-k}\left\|\wedge^{k+1} B\right\|^{s-k}\left\|\wedge^{k} A\right\|^{k+1-s}\left\|\wedge^{k} B\right\|^{k+1-s}=\varphi^{s}(A) \varphi^{s}(B)
\end{aligned}
$$

as claimed (where $\wedge^{k} A$ denotes the $k^{\text {th }}$ exterior power of $A$ ). It follows that each sequence $\left(a_{n}\right)$ defined by

$$
a_{n}:=\log \left(\sum_{i_{1}, \ldots, i_{n}=1}^{N} \varphi^{s}\left(A_{i_{1}} \cdots A_{i_{n}}\right)^{1-q} p_{i_{1}}^{q} \cdots p_{i_{n}}^{q}\right)
$$

satisfies $a_{n+m} \geq a_{n}+a_{m}$ for all $n, m \geq 1$, and this is well known to imply the convergence of the sequence $(1 / n) a_{n}$ to a limit in $(-\infty,+\infty]$. Observe that $\varphi^{s}(A) \geq$ $\sigma_{2}(A)^{s}$ for all $A \in M_{2}(\mathbf{R})$. Since $A_{1}, A_{2}$ are invertible we have $\sigma_{2}\left(A_{1}\right), \sigma_{2}\left(A_{2}\right) \geq \varepsilon$ for 
some $\varepsilon>0$, and thus

$$
\begin{aligned}
\sum_{i_{1}, \ldots, i_{n}=1}^{N} \varphi^{s}\left(A_{i_{1}} \cdots A_{i_{n}}\right)^{1-q} p_{i_{1}}^{q} \cdots p_{i_{n}}^{q} & \leq \sum_{i_{1}, \ldots, i_{n}=1}^{N} \sigma_{2}\left(A_{i_{1}} \cdots A_{i_{n}}\right)^{s(1-q)} p_{i_{1}}^{q} \cdots p_{i_{n}}^{q} \\
& \leq \sum_{i_{1}, \ldots, i_{n}=1}^{N} \varepsilon^{s(1-q)} p_{i_{1}}^{q} \cdots p_{i_{n}}^{q} \\
& =\varepsilon^{n s(1-q)}\left(p^{q}+(1-p)^{q}\right)^{n}
\end{aligned}
$$

(where we have used the fact that $1-q$ is negative) so that the limit is finite.

Let us show that $\mathbf{R}_{q}\left(A_{1}, A_{2}, p, s\right)$ is strictly increasing in $s$. We note that $\varphi^{s+t}(A)$ $\leq \varphi^{s}(A)\|A\|^{t}$ for all $s, t>0$ and for every matrix $A \in M_{2}(\mathbf{R})$. Taking $\theta:=$ $\max \left\{\left\|A_{1}\right\|,\left\|A_{2}\right\|\right\} \in(0,1)$ it follows that for all $n \geq 1$

$$
\sum_{i_{1}, \ldots, i_{n}=1}^{N} \varphi^{s+t}\left(A_{i_{1}} \cdots A_{i_{n}}\right)^{1-q} p_{i_{1}}^{q} \cdots p_{i_{n}}^{q} \geq \theta^{n t(1-q)} \sum_{i_{1}, \ldots, i_{n}=1}^{N} \varphi^{s}\left(A_{i_{1}} \cdots A_{i_{n}}\right)^{1-q} p_{i_{1}}^{q} \cdots p_{i_{n}}^{q}
$$

and therefore

$$
\mathbf{R}_{q}\left(A_{1}, A_{2}, p, s+t\right) \geq(1-q) t \log \theta+\mathbf{R}_{q}\left(A_{1}, A_{2}, p, s\right)>\mathbf{R}_{q}\left(A_{1}, A_{2}, p, s\right)
$$

as required.

Our interest in the previous lemma is due to the following consequence:

Corollary 2.2. For all invertible matrices $A_{1}, A_{2}$ such that $\left\|A_{1}\right\|,\left\|A_{2}\right\|<1$, all $p \in(0,1)$ and all $q>1$, we have

$$
\begin{aligned}
\mathfrak{r}_{q}\left(A_{1}, A_{2}, p\right) & =\sup \left\{s>0: \mathbf{R}_{q}\left(A_{1}, A_{2}, p, s\right)<0\right\} \\
& =\inf \left\{s>0: \mathbf{R}_{q}\left(A_{1}, A_{2}, p, s\right)>0\right\} .
\end{aligned}
$$

Before commencing the proof of Theorem 1.3, let us briefly describe its strategy. The proofs of continuity of the affinity dimension $\mathfrak{s}$ given in $[15,24]$ operate by defining the singular value pressure function

$$
\mathbf{S}\left(A_{1}, \ldots, A_{N}, s\right):=\lim _{n \rightarrow \infty} \frac{1}{n} \log \sum_{i_{1}, \ldots, i_{n}=1}^{N} \varphi^{s}\left(A_{i_{1}} \cdots A_{i_{n}}\right)
$$

and observing that for fixed invertible $A_{1}, \ldots, A_{N}$ with max $\left\|A_{i}\right\|<1$ the function $s \mapsto \mathbf{S}\left(A_{1}, \ldots, A_{N}, s\right)$ is strictly decreasing, so that

$$
\begin{aligned}
\mathfrak{s}\left(A_{1}, \ldots, A_{N}\right) & =\sup \left\{s>0: \mathbf{S}\left(A_{1}, \ldots, A_{N}, s\right)>0\right\} \\
& =\inf \left\{s>0: \mathbf{S}\left(A_{1}, \ldots, A_{N}, s\right)<0\right\} .
\end{aligned}
$$

The proofs then proceed by showing that for each fixed $s>0$ (or in the case of [24], for a dense set of $s>0)$ the function $\left(A_{1}, \ldots, A_{N}\right) \mapsto \mathbf{S}\left(A_{1}, \ldots, A_{N}, s\right)$ is continuous, and then deduce the continuity of $\mathfrak{s}$ via the formula $(2)$. The argument which we employ in proving Theorem 1.3 essentially converse to this: we demonstrate the existence of discontinuities in the function $\left(A_{1}, A_{2}\right) \mapsto \mathbf{R}_{q}\left(A_{1}, A_{2}, p, s\right)$ and show that they induce discontinuities in $\mathfrak{r}_{q}$ via the equation (1).

The origin of these discontinuities can be described informally as follows. Following [17], let us define the lower spectral radius of a pair of matrices $A_{1}, A_{2}$ to be the quantity

$$
\underline{\varrho}\left(A_{1}, A_{2}\right):=\lim _{n \rightarrow \infty} \min _{1 \leq i_{1}, \ldots, i_{n} \leq 2}\left\|A_{i_{1}} \cdots A_{i_{n}}\right\|^{\frac{1}{n}}=\inf _{n \geq 1} \min _{1 \leq i_{1}, \ldots, i_{n} \leq 2}\left\|A_{i_{1}} \cdots A_{i_{n}}\right\|^{\frac{1}{n}} .
$$


The lower spectral radius is known to depend discontinuously on the matrix entries in general [20, p. 20], and this phenomenon was investigated in depth by the author and Bochi in [7]. This relates to $\mathbf{R}_{q}\left(A_{1}, A_{2}, p, s\right)$ as follows: if $0<s \leq 1$ then we may estimate

$$
\begin{aligned}
\mathbf{R}_{q}\left(A_{1}, A_{2}, p, s\right) & \leq \limsup _{n \rightarrow \infty} \frac{1}{n} \log \left(\sum_{i_{1}, \ldots, i_{n}=1}^{2} p_{i_{1}}^{q} \cdots p_{i_{n}}^{q} \max _{1 \leq j_{1}, \ldots, j_{n} \leq 2}\left\|A_{j_{1}} \cdots A_{j_{n}}\right\|^{s(1-q)}\right) \\
& =\log \left(p^{q}+(1-p)^{q}\right)+s(1-q) \log \underline{\varrho}\left(A_{1}, A_{2}\right)
\end{aligned}
$$

-where the negativity of the exponent $s(1-q)$ has the critical effect of converting the maximum over all matrix products into a minimum - and similarly on the other hand

$$
\begin{aligned}
\mathbf{R}_{q}\left(A_{1}, A_{2}, p, s\right) & \geq \liminf _{n \rightarrow \infty} \frac{1}{n} \log \left(\min _{1 \leq i_{1}, \ldots, i_{n} \leq 2} p_{i_{1}}^{q} \cdots p_{i_{n}}^{q} \cdot \max _{1 \leq j_{1}, \ldots, j_{n} \leq 2}\left\|A_{j_{1}} \cdots A_{j_{n}}\right\|^{s(1-q)}\right) \\
& =q \log \min \{p, 1-p\}+s(1-q) \log \underline{\varrho}\left(A_{1}, A_{2}\right) .
\end{aligned}
$$

These estimates, despite their crudity, imply that if the discontinuity of the lower spectral radius $\varrho$ at a particular pair of matrices is strong enough then it induces a discontinuity in the function $\mathbf{R}_{q}$, which can be exploited to deduce a discontinuity in the function $\mathfrak{r}_{q}$. Indeed, the examples of discontinuity of $\mathfrak{r}_{q}$ in Theorem 1.3(i) and (ii) correspond directly with known examples of the discontinuity of the lower spectral radius, specifically Example 1.1 and Proposition 7.6 in [7]. In the context of Theorem 1.3(i) we can obtain sufficient control on the size of the discontinuity in $\underline{\varrho}$ without any assumptions on $p$ and $q$. In the context of Theorem 1.3(ii) our much weaker control on the discontinuities of $\varrho$ means that the above estimate is only useful if $q$ is large and $p$ is close to $\frac{1}{2}$, which has the effect of bringing the quantities $\log \left(p^{q}+(1-p)^{q}\right)$ and $q \log (\min \{p,(1-p)\})$ closer together. In order to deal with more general $p$ the proof of Theorem 1.3(ii) in fact applies a slightly finer estimate than that indicated above: for this we require a slightly strengthened statement of [7, Proposition 7.6], which shows not only that the lower spectral radius is discontinuous in certain places but also specifies how it is discontinuous. We nonetheless emphasise that the conceptual origin of the discontinuity of $\mathfrak{r}_{q}$ in this paper is that it is a consequence of the discontinuity of the lower spectral radius.

Proof of Theorem 1.3(i). Let $q>1$ and $0<\lambda<\frac{1}{2}$, and let $A_{1}, A_{2}, \delta$ be as in Theorem 1.3(i). Throughout the proof we shall find it useful to write $p_{1}:=p$, $p_{2}:=(1-p)$ in order to simplify certain frequently-arising expressions. We observe that by straightforward differentiation and minimisation with respect to $p$ one has $p^{q}+(1-p)^{q} \geq 2^{1-q}$ for every $q>1$ and $p \in(0,1)$. In particular, noting the hypothesis of Theorem 1.3(i) and the negativity of $\log \lambda$,

$$
0<\frac{q \log \min \{p, 1-p\}}{(q-1) \log \sqrt{\lambda \delta}}<\frac{\log \left(p^{q}+(1-p)^{q}\right)}{(q-1) \log \lambda} \leq \frac{\log \frac{1}{2}}{\log \lambda}<1 .
$$

Clearly $\left\|A_{i_{1}} \cdots A_{i_{n}}\right\|=\lambda^{n}$ for every $i_{1}, \ldots, i_{n} \in\{1,2\}$ and $n \geq 1$, so for every $s \in(0,1]$

$$
\begin{aligned}
\mathbf{R}_{q}\left(A_{1}, A_{2}, p, s\right) & =\lim _{n \rightarrow \infty} \frac{1}{n} \log \left(\sum_{i_{1}, \ldots, i_{n}=1}^{2} \lambda^{s n(1-q)} p_{i_{1}}^{q} \cdots p_{i_{n}}^{q}\right) \\
& =s(1-q) \log \lambda+\log \left(p^{q}+(1-p)^{q}\right) .
\end{aligned}
$$


In particular if $s<\log \left(p^{q}+(1-p)^{q}\right) /(q-1) \log \lambda \in(0,1]$ then $\mathbf{R}_{q}\left(B_{1}, B_{2}, p, s\right)<0$, so we have

$$
\mathfrak{r}_{q}\left(A_{1}, A_{2}, p\right) \geq \frac{\log \left(p^{q}+(1-p)^{q}\right)}{(q-1) \log \lambda}
$$

using Corollary 2.2. Now fix an integer $k \geq 1$ and define $B_{1}:=A_{1}$ and

$$
B_{2}:=\lambda\left(\begin{array}{cc}
\cos \frac{\pi}{2 k} & -\sin \frac{\pi}{2 k} \\
\sin \frac{\pi}{2 k} & \cos \frac{\pi}{2 k}
\end{array}\right)
$$

so that

$$
B_{2}^{k}=\left(\begin{array}{cc}
0 & -\lambda^{k} \\
\lambda^{k} & 0
\end{array}\right)
$$

(We observe that by taking $k$ sufficiently large, $\left(B_{1}, B_{2}\right)$ may be taken as close to $\left(A_{1}, A_{2}\right)$ as desired.) Since we have

$$
B_{1}^{n} B_{2}^{k} B_{1}^{n}=\left(\begin{array}{cc}
\lambda^{n} & 0 \\
0 & \delta^{n}
\end{array}\right)\left(\begin{array}{cc}
0 & -\lambda^{k} \\
\lambda^{k} & 0
\end{array}\right)\left(\begin{array}{cc}
\lambda^{n} & 0 \\
0 & \delta^{n}
\end{array}\right)=\left(\begin{array}{cc}
\lambda^{n+k} \delta^{n} & 0 \\
0 & \lambda^{n+k} \delta^{n}
\end{array}\right)
$$

for all $n \geq 1$ it follows that

$$
\min _{1 \leq i_{1}, \ldots, i_{2 n+k} \leq 2}\left\|B_{i_{1}} \cdots B_{i_{n}}\right\| \leq \lambda^{n+k} \delta^{n}
$$

for every $n \geq 1$, and therefore

$$
\lim _{n \rightarrow \infty}\left(\min _{1 \leq i_{1}, \ldots, i_{n} \leq 2}\left\|B_{i_{1}} \cdots B_{i_{n}}\right\|\right)^{\frac{1}{n}} \leq \sqrt{\lambda \delta} .
$$

Hence for $0<s \leq 1$

$$
\begin{aligned}
& \mathbf{R}_{q}\left(B_{1}, B_{2}, p, s\right)=\lim _{n \rightarrow \infty} \frac{1}{n} \log \left(\sum_{i_{1}, \ldots, i_{n}=1}^{2}\left\|B_{i_{1}} \cdots B_{i_{n}}\right\|^{s(1-q)} p_{i_{1}}^{q} \cdots p_{i_{n}}^{q}\right) \\
& \geq \lim _{n \rightarrow \infty} \frac{1}{n} \log \left(\max _{1 \leq i_{1}, \ldots, i_{n} \leq 2}\left(\left\|B_{i_{1}} \cdots B_{i_{n}}\right\|^{s(1-q)}\right) \min \left\{p^{q n},(1-p)^{q n}\right\}\right) \\
& =\lim _{n \rightarrow \infty} \frac{1}{n} \log \left(\left(\min _{1 \leq i_{1}, \ldots, i_{n} \leq 2}\left\|B_{i_{1}} \cdots B_{i_{n}}\right\|\right)^{s(1-q)} \min \left\{p^{q n},(1-p)^{q n}\right\}\right) \\
& \geq \frac{s(1-q)}{2} \log (\lambda \delta)+\log \min \left\{p^{q},(1-p)^{q}\right\} .
\end{aligned}
$$

If $1 \geq s>q \log \min \{p, 1-p\} /(q-1) \log \sqrt{\lambda \delta} \in(0,1)$ then this last term exceeds 0 and therefore $\mathfrak{r}_{q}\left(B_{1}, B_{2}, p\right) \geq s$. Hence in view of Corollary 2.2

$$
\mathfrak{r}_{q}\left(B_{1}, B_{2}, p\right) \leq \frac{q \log \min \{p, 1-p\}}{(q-1) \log \sqrt{\lambda \delta}} .
$$

As was previously noted, by taking the integer $k$ in the definition of $B_{2}$ arbitrarily large we may take $\left(B_{1}, B_{2}\right)$ as above arbitrarily close to $\left(A_{1}, A_{2}\right)$, and it follows that

$$
\begin{aligned}
\liminf _{\left(B_{1}, B_{2}\right) \rightarrow\left(A_{1}, A_{2}\right)} \mathfrak{r}_{q}\left(B_{1}, B_{2}, p\right) & \leq \frac{2 q \log \min \{p, 1-p\}}{(q-1) \log (\lambda \delta)} \\
& <\frac{\log \left(p^{q}+(1-p)^{q}\right)}{(q-1) \log \lambda} \leq \mathfrak{r}_{q}\left(A_{1}, A_{2}, p\right)
\end{aligned}
$$

where we have used (3), so that $\mathfrak{r}_{q}$ is discontinuous at $\left(A_{1}, A_{2}\right)$ as claimed. This completes the proof of (i). 
The proof of (ii) uses closely analogous estimates, but we require an additional result relating to Conjecture 1.2. The following result is a more specialised reworking of one half of [7, Proposition 7.6].

Lemma 2.3. Suppose that Conjecture 1.2 is true. Then there exist $\varepsilon, \kappa>0$ and a set $X \subset M_{2}(\mathbf{R})^{2}$ with positive Lebesgue measure such that for all $\left(A_{1}, A_{2}\right) \in X$ we have

$$
\left\|A_{1}\right\|,\left\|A_{2}\right\|<\frac{1}{2}, \quad \underline{\varrho}\left(A_{1}, A_{2}\right) \geq e^{-\kappa},
$$

but such that in every open neighbourhood of $\left(A_{1}, A_{2}\right)$ we may find $\left(B_{1}, B_{2}\right)$ such that for a certain integer $k \geq 1$ depending on $\left(B_{1}, B_{2}\right)$

$$
\lim _{n \rightarrow \infty}\left\|B_{1}^{n} B_{2}^{k} B_{1}^{n}\right\|^{\frac{1}{2 n+k}}=\underline{\varrho}\left(B_{1}, B_{2}\right) \leq e^{-\varepsilon-\kappa} .
$$

Proof. Let us first define

$$
Z:=\left\{(\alpha H, \beta R): H \in \mathcal{H}, R \in \mathcal{E} \text { and } 0<\alpha<\beta<\frac{1}{2\|H\|}\right\}
$$

Clearly this is an open subset of $M_{2}(\mathbf{R})^{2}$, and for every $\left(A_{1}, A_{2}\right) \in Z$ both of the matrices $A_{i}$ have positive determinant and have norm strictly less than one half. By the hypothesis that Conjecture 1.2 is true, the set of all $(\alpha H, \beta R) \in Z$ such that $(H, R)$ is resistant has full Lebesgue measure in $Z$, and hence in particular has positive Lebesgue measure in $M_{2}(\mathbf{R})^{2}$.

We first claim that for all $\left(A_{1}, A_{2}\right)=(\alpha H, \beta R)$ such that $(H, R)$ is resistant we have $\varrho\left(A_{1}, A_{2}\right)>\sqrt{\operatorname{det} A_{1}}$. Indeed, suppose that $(H, R)$ is $(c, \lambda, \varepsilon)$-resistant where $c, \varepsilon>0$ and $\lambda>1$. If $A_{i_{1}}, \ldots, A_{i_{n}}$ contains at most $\varepsilon n$ instances of $A_{2}$ then we have

$$
\left\|A_{i_{1}} \cdots A_{i_{n}}\right\| \geq c \lambda^{n} \alpha^{n}
$$

since $\left\|A_{i_{1}} \cdots A_{i_{n}}\right\|$ is at least $\alpha^{n}$ times the norm of a product of $n$ of the matrices $H, R$ in which at most $\varepsilon n$ matrices are equal to $R$. On the other hand if the product $A_{i_{1}} \cdots A_{i_{n}}$ contains at least $\varepsilon n$ instances of $A_{2}$ then we have

$$
\left\|A_{i_{1}} \cdots A_{i_{n}}\right\| \geq \sqrt{\left|\operatorname{det} A_{i_{1}} \cdots A_{i_{n}}\right|} \geq \alpha^{(1-\varepsilon) n} \beta^{\varepsilon n}
$$

and therefore

$$
\underline{\varrho}\left(A_{1}, A_{2}\right)=\lim _{n \rightarrow \infty} \inf _{1 \leq i_{1}, \ldots, i_{n} \leq 2}\left\|A_{i_{1}} \cdots A_{i_{n}}\right\|^{\frac{1}{n}} \geq \min \left\{\lambda \alpha,\left(\frac{\beta}{\alpha}\right)^{\varepsilon} \alpha\right\}>\alpha=\sqrt{\operatorname{det} A_{1}}
$$

as claimed.

We next claim that for every $\left(A_{1}, A_{2}\right)=(\alpha H, \beta R) \in Z$, we may in every open neighbourhood of $A_{2}$ find a matrix $B_{2}$ such that for some integer $k \geq 1$ depending on $B_{2}$,

$$
\lim _{n \rightarrow \infty}\left\|A_{1}^{n} B_{2}^{k} A_{1}^{n}\right\|^{\frac{1}{2 n+k}}=\sqrt{\operatorname{det} A_{1}} .
$$

(We note that in this case necessarily $\varrho\left(A_{1}, B_{2}\right)=\sqrt{\operatorname{det} A_{1}}$, since clearly any product of $n$ of those two matrices is bounded below in norm by the square root of the determinant, which in turn is bounded below by $\left(\operatorname{det} A_{1}\right)^{n / 2}$.) To show this it is sufficient to show that for any fixed $H \in \mathcal{H}$ and $R \in \mathcal{E}$, we may in every open neighbourhood of $R$ find a matrix $R^{\prime}$ such that for some integer $k \geq 1$ depending on $R^{\prime}$,

$$
\lim _{n \rightarrow \infty}\left\|H^{n}\left(R^{\prime}\right)^{k} H^{n}\right\|^{\frac{1}{2 n+k}}=1
$$


Let us prove this statement. Given $(H, R) \in \mathcal{H} \times \mathcal{E}$ let $\lambda>1$ denote the larger eigenvalue of $H$, and let $u$ and $v$ be eigenvectors of $H$ corresponding respectively to the eigenvalues $\lambda$ and $\lambda^{-1}$. Since $R$ has non-real eigenvalues it is conjugate to a rotation through some particular angle $\theta$. It is easy to see that this implies that in any neighbourhood of $R$ we may find a matrix $R^{\prime}$, conjugate to a rotation through a different angle, such that $\left(R^{\prime}\right)^{k} u=\gamma v$ for some nonzero real number $\gamma$ and integer $k \geq 1$. In the basis $(u, v)$ we have

$$
H^{n}\left(R^{\prime}\right)^{k} H^{n}=\left(\begin{array}{cc}
\lambda^{n} & 0 \\
0 & \lambda^{-n}
\end{array}\right)\left(\begin{array}{cc}
0 & \delta \\
\gamma & \epsilon
\end{array}\right)\left(\begin{array}{cc}
\lambda^{n} & 0 \\
0 & \lambda^{-n}
\end{array}\right)=\left(\begin{array}{cc}
0 & \delta \\
\gamma & \lambda^{-2 n} \epsilon
\end{array}\right)
$$

for some real numbers $\delta, \epsilon$, where the first column of $\left(R^{\prime}\right)^{k}$ reflects the fact that $\left(R^{\prime}\right)^{k} u=\gamma v$. In particular $\left\|H^{n}\left(R^{\prime}\right)^{k} H^{n}\right\|$ is bounded independently of $n$, and this yields (4).

Summarising the proof so far, we have shown that there is a full-measure subset $Z_{1}$ of the open set $Z \subset M_{2}(\mathbf{R})^{2}$ such that every $\left(A_{1}, A_{2}\right) \in Z_{1}$ satisfies $\left\|A_{1}\right\|,\left\|A_{2}\right\|<\frac{1}{2}$ and $\underline{\varrho}\left(A_{1}, A_{2}\right)>\sqrt{\operatorname{det} A_{1}}$, and has the property that in every open neighbourhood of $A_{2}$ we may find $B_{2}$ such that for some integer $k \geq 1$,

$$
\lim _{n \rightarrow \infty}\left\|A_{1}^{n} B_{2}^{k} A_{1}^{n}\right\|^{\frac{1}{2 n+k}}=\underline{\varrho}\left(A_{1}, B_{2}\right)=\sqrt{\operatorname{det} A_{1}} .
$$

So, let us choose $\kappa>0$ such that the set

$$
Z_{2}:=\left\{\left(A_{1}, A_{2}\right) \in Z_{1}: \underline{\varrho}\left(A_{1}, A_{1}\right) \geq e^{-\kappa}>\sqrt{\operatorname{det} A_{1}}\right\}
$$

has positive Lebesgue measure, and choose $\varepsilon>0$ such that the set

$$
X:=\left\{\left(A_{1}, A_{2}\right) \in Z_{2}: \sqrt{\operatorname{det} A_{1}}<e^{-\kappa-\varepsilon}\right\}
$$

has positive Lebesgue measure. The proof is complete.

Remark. In order to improve Theorem 1.3(ii) so as to allow arbitrary $q>1$ it would be sufficient to be able to choose the set $X$ in such a way that the ratio $\varepsilon / \kappa$ is made arbitrarily large. In effect, this asks that we should be able to reduce the second singular value of $A_{1}$ arbitrarily far without simultaneously reducing $\varrho\left(A_{1}, A_{2}\right)$ by a comparable amount: in Theorem 1.3(i), this effect is achieved by the simple expedient of reducing $\delta$.

Proof of Theorem 1.3(ii). Let $X, \varepsilon, \kappa$ be as in Lemma 2.3 and choose $Q:=$ $1+\frac{\kappa}{\varepsilon}>1$. For all $p \in\left[\frac{1}{2}, 1\right)$ and $q>Q$ we have

$$
\left(p^{q}+(1-p)^{q}\right)^{\frac{1}{q}}<p^{\frac{\kappa}{\kappa+\varepsilon}}
$$

since for each fixed $q>1$ the former expression is a convex function of $p$, the latter is a concave function of $p$, the two functions agree at $p=1$ and the former function is strictly less than the latter at $p=\frac{1}{2}$. Rearranging we find that for all such $p$ and $q$

$$
\frac{\log p^{q}}{\log \left(p^{q}+(1-p)^{q}\right)}<1+\frac{\varepsilon}{\kappa} .
$$

Since clearly $e^{-\kappa} \leq \underline{\varrho}\left(A_{1}, A_{2}\right) \leq \max \left\{\left\|A_{1}\right\|,\left\|A_{2}\right\|\right\}<\frac{1}{2}$ we have $\kappa>\log 2$ and therefore

$$
0<\frac{\log \left(p^{q}\right)}{(1-q)(\varepsilon+\kappa)}<\frac{\log \left(p^{q}+(1-p)^{q}\right)}{(1-q) \kappa}<\frac{\log \left(p^{q}+(1-p)^{q}\right)}{(1-q) \log 2} \leq 1
$$


for all $p \in\left[\frac{1}{2}, 1\right)$ and $q>Q$, where we have reused the elementary inequality $p^{q}+(1-$ $p)^{q} \geq 2^{1-q}$ which was similarly applied in (i). We will show that for all such $p$ and $q$, every point of $X$ is a point of discontinuity of the map $\left(A_{1}, A_{2}\right) \mapsto \mathfrak{r}_{q}\left(A_{1}, A_{2}, p\right)$.

Let us therefore fix $p$ and $q$ and take $\left(A_{1}, A_{2}\right) \in X$. For every $s \in(0,1]$ we have

$$
\begin{aligned}
\mathbf{R}_{q}\left(A_{1}, A_{2}, p, s\right) & =\lim _{n \rightarrow \infty} \frac{1}{n} \log \left(\sum_{i_{1}, \ldots, i_{n}=1}^{2}\left\|A_{i_{1}} \cdots A_{i_{n}}\right\|^{s(1-q)} p_{i_{1}}^{q} \cdots p_{i_{n}}^{q}\right) \\
& \leq \lim _{n \rightarrow \infty} \frac{1}{n} \log \left(\max _{1 \leq i_{1}, \ldots, i_{n} \leq 2}\left(\left\|A_{i_{1}} \cdots A_{i_{n}}\right\|^{s(1-q)}\right) \sum_{j_{1}, \ldots, j_{n}=1}^{2} p_{j_{1}}^{q} \cdots p_{j_{n}}^{q}\right) \\
& \left.=\lim _{n \rightarrow \infty} \frac{1}{n} \log \left(\min _{1 \leq i_{1}, \ldots, i_{n} \leq 2}\left\|A_{i_{1}} \cdots A_{i_{n}}\right\|\right)^{s(1-q)} \sum_{j_{1}, \ldots, j_{n}=1}^{2} p_{j_{1}}^{q} \cdots p_{j_{n}}^{q}\right) \\
& =s(1-q) \log \underline{\varrho}\left(A_{1}, A_{2}\right)+\log \left(p^{q}+(1-p)^{q}\right) \\
& \leq s(q-1) \kappa+\log \left(p^{q}+(1-p)^{q}\right) .
\end{aligned}
$$

It follows that if $s<\log \left(p^{q}+(1-p)^{q}\right) /(1-q) \kappa<1$ then $\mathbf{R}_{q}\left(A_{1}, A_{2}, p, s\right)$ is negative, and hence by Corollary 2.2

$$
\mathfrak{r}_{q}\left(A_{1}, A_{2}, p\right) \geq \frac{\log \left(p^{q}+(1-p)^{q}\right)}{(1-q) \kappa} .
$$

On the other hand we may take $\left(B_{1}, B_{2}\right)$ arbitrarily close to $\left(A_{1}, A_{2}\right)$ such that

$$
\lim _{n \rightarrow \infty}\left\|B_{1}^{n} B_{2}^{k} B_{1}^{n}\right\|^{\frac{1}{2 n+k}}=\underline{\varrho}\left(B_{1}, B_{2}\right)<e^{-\varepsilon-\kappa}
$$

for some integer $k \geq 1$. In particular we have

$$
\begin{aligned}
& \lim _{n \rightarrow \infty} \frac{1}{n} \log \max _{1 \leq i_{1}, \ldots, i_{n} \leq 2}\left(\left\|B_{i_{1}} \cdots B_{i_{n}}\right\|^{s(1-q)} p_{i_{1}}^{q} \cdots p_{i_{n}}^{q}\right) \\
& \leq \lim _{n \rightarrow \infty} \frac{1}{n} \log \max _{1 \leq i_{1}, \ldots, i_{n} \leq 2}\left(\left\|B_{i_{1}} \cdots B_{i_{n}}\right\|\right)^{s(1-q)}+\lim _{n \rightarrow \infty} \frac{1}{n} \log \max _{1 \leq i_{1}, \ldots, i_{n} \leq 2}\left(p_{i_{1}}^{q} \cdots p_{i_{n}}^{q}\right) \\
& =\lim _{n \rightarrow \infty} \frac{s(1-q)}{n} \log \min _{1 \leq i_{1}, \ldots, i_{n} \leq 2}\left\|B_{i_{1}} \cdots B_{i_{n}}\right\|+q \log p \\
& =s(1-q) \log \underline{\varrho}\left(B_{1}, B_{2}\right)+q \log p
\end{aligned}
$$

since $p_{1}:=p \geq \frac{1}{2} \geq 1-p=p_{2}$, but also

$$
\begin{aligned}
& \lim _{n \rightarrow \infty} \frac{1}{n} \log \max _{1 \leq i_{1}, \ldots, i_{n} \leq 2}\left(\left\|B_{i_{1}} \cdots B_{i_{n}}\right\|^{s(1-q)} p_{i_{1}}^{q} \cdots p_{i_{n}}^{q}\right) \\
& \geq \lim _{n \rightarrow \infty} \frac{1}{2 n+k} \log \left(\left\|B_{1}^{n} B_{2}^{k} B_{1}^{n}\right\|^{s(1-q)} p_{1}^{n} p_{2}^{k} p_{1}^{n}\right) \\
& =s(q-1) \log \underline{\varrho}\left(B_{1}, B_{2}\right)+q \log p,
\end{aligned}
$$

and we conclude that the limit

$$
\lim _{n \rightarrow \infty} \frac{1}{n} \log \max _{1 \leq i_{1}, \ldots, i_{n} \leq 2}\left(\left\|B_{i_{1}} \cdots B_{i_{n}}\right\|^{s(1-q)} p_{i_{1}}^{q} \cdots p_{i_{n}}^{q}\right)
$$


is equal to $s(q-1) \log \underline{\varrho}\left(B_{1}, B_{2}\right)+q \log p$. It follows that for all $s \in(0,1]$

$$
\begin{aligned}
\mathbf{R}_{q}\left(B_{1}, B_{2}, p, s\right) & =\lim _{n \rightarrow \infty} \frac{1}{n} \log \left(\sum_{i_{1}, \ldots, i_{n}=1}^{2}\left\|B_{i_{1}} \cdots B_{i_{n}}\right\|^{s(1-q)} p_{i_{1}}^{q} \cdots p_{i_{n}}^{q}\right) \\
& \geq \lim _{n \rightarrow \infty} \frac{1}{n} \log \left(\max _{1 \leq i_{1}, \ldots, i_{n} \leq 2}\left(\left\|B_{i_{1}} \cdots B_{i_{n}}\right\|^{s(1-q)} p_{i_{1}}^{q} \cdots p_{i_{n}}^{q}\right)\right) \\
& =s(1-q) \log \underline{\varrho}\left(B_{1}, B_{2}\right)+q \log p \\
& >s(q-1)(\varepsilon+\kappa)+q \log p .
\end{aligned}
$$

If $1 \geq s>(q \log p) /(1-q)(\varepsilon+\kappa) \in(0,1)$ then $\mathbf{R}_{q}\left(B_{1}, B_{2}, p, s\right)>0$, and therefore

by Corollary 2.2. Hence

$$
\mathfrak{r}_{q}\left(B_{1}, B_{2}, p\right) \leq \frac{\log \left(p^{q}\right)}{(1-q)(\varepsilon+\kappa)}
$$

$$
\begin{aligned}
\liminf _{\left(B_{1}, B_{2}\right) \rightarrow\left(A_{1}, A_{2}\right)} \mathfrak{r}_{q}\left(B_{1}, B_{2}, p\right) & \leq \frac{q \log p}{(1-q)(\varepsilon+\kappa)} \\
& <\frac{\log \left(p^{q}+(1-p)^{q}\right)}{(1-q) \kappa} \\
& \leq \mathfrak{r}_{q}\left(A_{1}, A_{2}, p\right)
\end{aligned}
$$

using (5), and $\left(B_{1}, B_{2}\right) \mapsto \mathfrak{r}_{q}\left(B_{1}, B_{2}, p\right)$ is discontinuous at $\left(A_{1}, A_{2}\right)$ as claimed.

Acknowledgements. The author was supported by EPSRC grant EP/L026953/1.

\section{References}

[1] Allen, J., B. Seeger, and D. Unger: On the size of the resonant set for the products of $2 \times 2$ matrices. Involve 4:2, 2011, 157-166.

[2] Avila, A., and T. Roblin: Uniform exponential growth for some $\mathrm{SL}(2, \mathbf{R})$ matrix products. - J. Mod. Dyn. 3:4, 2009, 549-554.

[3] Barański, K.: Hausdorff dimension of the limit sets of some planar geometric constructions. - Adv. Math., 210:1, 2007, 215-245.

[4] Barral, J., and D.-J. FEnG: Multifractal formalism for almost all self-affine measures. Comm. Math. Phys. 318:2, 2013, 473-504.

[5] Bedford, T.: Crinkly curves, Markov partitions and box dimensions in self-similar sets. PhD thesis, University of Warwick, 1984.

[6] Bochi, J., and B. FAYAD: Dichotomies between uniform hyperbolicity and zero Lyapunov exponents for SL(2, R) cocycles. - Bull. Braz. Math. Soc. (N.S.) 37:3, 2006, 307-349.

[7] Bochi, J., and I. D. Morris: Continuity properties of the lower spectral radius. - Proc. Lond. Math. Soc. (3) 110:2, 2015, 477-509.

[8] Falconer, K. J.: The Hausdorff dimension of self-affine fractals. - Math. Proc. Cambridge Philos. Soc. 103:2, 1988, 339-350.

[9] Falconer, K. J.: Techniques in fractal geometry. - John Wiley \& Sons, Ltd., Chichester, 1997.

[10] FAlconer, K. J.: Generalized dimensions of measures on self-affine sets. - Nonlinearity 12:4, 1999, 877-891.

[11] FAlconer, K. J.: Fractal geometry: mathematical foundations and applications. Second edition. - John Wiley \& Sons, Inc., Hoboken, NJ, 2003.

[12] FALCONER, K. J.: Generalized dimensions of measures on almost self-affine sets. - Nonlinearity $23: 5,2010,1047-1069$. 
[13] Falconer, K. J.: Dimensions of self-affine sets: a survey. - In: Further developments in fractals and related fields, Trends Math., Birkhäuser/Springer, New York, 2013, 115-134.

[14] FAYAd, B., and R. KRIKorian: Exponential growth of product of matrices in $\operatorname{SL}(2, \mathbf{R})$. Nonlinearity 21:2, 2008, 319-323.

[15] Feng, D.-J., and P. Shmerkin: Non-conformal repellers and the continuity of pressure for matrix cocycles. - Geom. Funct. Anal. 24:4, 2014, 1101-1128.

[16] Fraser, J. M.: On the packing dimension of box-like self-affine sets in the plane. - Nonlinearity 25:7, 2012, 2075-2092.

[17] GuRvits, L.: Stability of discrete linear inclusion. - Linear Algebra Appl. 231, 1995, 47-85.

[18] Hutchinson, J. E.: Fractals and self-similarity. - Indiana Univ. Math. J. 30:5, 1981, 713-747.

[19] Jordan, T., and M. Pollicott, and K. Simon: Hausdorff dimension for randomly perturbed self affine attractors. - Comm. Math. Phys. 270:2, 2007, 519-544.

[20] Jungers, R.: The joint spectral radius: theory and applications. - Lecture Notes in Control and Inform. Sci. 385, Springer-Verlag, Berlin, 2009.

[21] Lalley, S. P., and D. Gatzouras: Hausdorff and box dimensions of certain self-affine fractals. - Indiana Univ. Math. J. 41:2, 1992, 533-568.

[22] LAU, K.-S.: Self-similarity, $L^{p}$-spectrum and multifractal formalism. - In: Fractal geometry and stochastics (Finsterbergen, 1994), Progr. Probab. 37, Birkhäuser, Basel, 1995, 55-90.

[23] McMullen, C.: The Hausdorff dimension of general Sierpiński carpets. - Nagoya Math. J. 96, 1984, 1-9.

[24] Morris, I. D.: An inequality for the matrix pressure function and applications. - Adv. Math. 302, 2016, 280-308.

[25] Solomyak, B.: Measure and dimension for some fractal families. - Math. Proc. Cambridge Philos. Soc. 124:3, 1998, 531-546.

Received 29 October 2015 • Accepted 5 August 2016 